\title{
Telomere-Mitochondrion Links Contribute to Induction of Senescence in MCF-7 Cells after Carbon-Ion Irradiation
}

\author{
Guo-Ying Miao ${ }^{1,2,3,4,5}$, Xin Zhou ${ }^{1,2,3}$, Xin Zhang ${ }^{1,2,3}$, Yi Xie ${ }^{1,2,3}$, Chao Sun ${ }^{1,2,3}$, Yang \\ Liu $^{1,2,3}$, Lu Gan ${ }^{1,2,3}$, Hong Zhang ${ }^{1,2,3 *}$
}

\begin{abstract}
The effects of carbon-ion irradiation on cancer cell telomere function have not been comprehensively studied. In our previous report cancer cells with telomere dysfunction were more sensitive to carbon-ion irradiation, but the underlying mechanisms remained unclear. Here we found that telomerase activity was suppressed by carbon-ion irradiation via hTERT down-regulation. Inhibition of telomere activity by MST-312 further increased cancer cell radiosensitivity to carbon-ion radiation. hTERT suppression caused by either carbon-ion irradiation or MST-312 impaired mitochondrial function, as indicated by decreased membrane potential, mtDNA copy number, mitochondrial mass, total ATP levels and elevated reactive oxygen species (ROS). PGC-1 $\alpha$ expression was repressed after carbion-ion irradiation, and hTERT inhibition by MST-312 could further exacerbate this effect. Lowering the mitochondrial ROS level by MitoTEMPO could partially counteract the induction of cellular senescence induced by carbon-ion radiation and MST-312 incubation. Taken together, the current data suggest that telomere-mitochondrion links play a role in the induction of senescence in MCF-7 cells after carbon-ion irradiation.
\end{abstract}

Keywords: Telomere - mitochondrion - senescence - carbon-ion irradiation

Asian Pac J Cancer Prev, 17 (4), 1993-1998

\section{Introduction}

Heavy-ion radiotherapy has demonstrated the advantages over conventional radiotherapy, taking advantage of its improved dose conformation and higher relative biological effectiveness (Jakel et al., 2008). Telomerase is active in about $90 \%$ cancer cells which is important for cancer cells to maintain their immortalization. But the effects of heavy-ion radiation on telomere are largely unknown. Understanding how telomere influences sensitivity to carbon-ion is highly relevant given the emergence of telomerase inhibition as a potential cancer therapeutic modality. Currently few reports have investigated the effects of heavy-ion on telomere function. Durante et al. (2006) reported loss of telomere in the progeny of human lymphocytes after exposure to heavy-ion. Our previous findings showed that telomere function is closely related to carbon-ion radiation-induced cancer cell inactivation (Zhou et al., 2013), but the missing link between telomere dysfunction and cancer cell death remain elusive.

Our previous paper found that carbon-ion radiation induced extensive senescence in breast cancer cells MCF-7 (Zhou et al., 2013). MCF-7 senescence has been reported to be caused by excessive intracellular reactive oxygen species (ROS) generation (Li et al., 2013; Lee et al., 2014). Mitochondria are the main source of intracellular ROS within cells that may contribute to the ROSmediated senescence process. Interestingly, the human catalytic subunit of the telomere that works as a reverse transcriptase (human telomerase reverse transcriptase, hTERT) is reversibly excluded from the nucleus (Santos et al., 2006b) and localizes in mitochondria (Buchner et al., 2010). 10-20\% of the total cellular content of hTERT is mitochondrial under normal conditions (Buchner et al., 2010; Haendeler et al., 2009), while $80 \%$ of the protein could accumulate in a time-dependent manner in mitochondria under oxidative stress (Ahmed et al., 2008). The accumulation of hTERT within mitochondria protects mitochondria under oxidative stress, as indicated by increased mitochondrial membrane potential and decreased cell peroxide levels and mitochondrial superoxide production (Ahmed et al., 2008). The protective effects of mt-hTERT on mitochondria have been extensively reviewed (Saretzki, 2009; Gordon and Santos, 2010; Indran et al., 2011). Thus we suggest that the different radiosensitivity of MCF-7 cells with/without telomere dysfunction could be caused by bidirectional

${ }^{I}$ Department of Heavy Ion Radiation Medicine, Institute of Modern Physics, Chinese Academy of Sciences, ${ }^{2}$ Key Laboratory of Heavy Ion Radiation Biology and Medicine of Chinese Academy of Sciences, ${ }^{3}$ Key Laboratory of Heavy Ion Radiation Medicine of Gansu Province, ${ }^{4}$ Gansu Provincial Hospital, Lanzhou, Gansu, ${ }^{5}$ Graduate School of Chinese Academy of Sciences, Beijing, China *For correspondence: zhangh@impcas.ac.cn 
interactions between telomere and mitochondria (Liu et al., 2002; Sahin et al., 2011).

In the present study, we found that hTERT was down-regulated both in transcript and expression level in MCF-7 cells after carbon-ion irradiation. Furthermore, telomerase inhibitor MST-312 could greatly increase MCF-7 radiosensitivity to carbon-ion. MST-312 treatment alone caused mitochondria dysfunction, while carbon-ion irradiation could exacerbate this effect. Mitochondrial ROS scavenger MitoTEMPO partially counteracts the induction of cellular senescence after carbon-ion radiation. In conclusion, our data provide evidences supporting that telomere-mitochondrion link play a role in the inactivation process of cancer cells exposure to carbon-ion irradiation.

\section{Materials and Methods}

\section{Cell culture and irradiation treatment}

The human breast cancer cell line MCF-7 was purchased from the American Type Culture Collection. Cells were maintained in Dulbecco's Modified Eagle's Medium (Gibco, USA) supplemented with $10 \%$ fetal bovine serum. Cells were cultured in $5 \% \mathrm{CO} 2$ in humidified air at $37^{\circ} \mathrm{C}$. For MST-312 treatment, cells were continuously incubated with $2 \mu \mathrm{M}$ MST312 (Sigma,USA) for 30-70 days in Dulbecco's Modified Eagle's Medium (Gibco, USA) supplemented with 10\% fetal bovine serum. The medium with $2 \mu \mathrm{M}$ MST312 was replaced every 3 to 4 days.

Carbon-ion irradiations were performed at room temperature at the Heavy Ion Medical Accelerator Center (HIMAC) of the National Institute of Radiological Sciences (Chiba, Japan), with $290 \mathrm{MeV} / \mathrm{n}$ carbon-ion; the LET value for carbon-ion was $40 \mathrm{KeV} / \mu \mathrm{m}$. The dose rates were $1 \mathrm{~Gy} / \mathrm{min}$.

\section{Telomerase assay}

Telomerase activity was measured using the TeloTAGGG Telomerase PCR ELISAplus kit (Roche Diagnostics, USA) according to manufacturer's instructions. In brief, cells were resuspended in $200 \mu \mathrm{l}$ lysis reagent and incubated for $30 \mathrm{~min}$ on ice. $2 \mu \mathrm{l}$ of the cell extract (corresponding to $2 \times 10^{4}$ cell equivalents) was added to an elongation/amplification mixture composed of $25 \mu 12 \mathrm{x}$ reaction mixture and $5 \mu 1$ of internal standard (IS) (both supplied in the kit) in a total volume of $50 \mu 1$. A combined primer elongation/amplification reaction was performed according to the following protocol: elongation at $23^{\circ} \mathrm{C}$ for $20 \mathrm{~min}$, telomerase inactivation at $94^{\circ} \mathrm{C}$ for $5 \mathrm{~min}$ and 30 times $94^{\circ} \mathrm{C}$ for $30 \mathrm{sec}, 50^{\circ} \mathrm{C}$ for $30 \mathrm{sec}$ and $72^{\circ} \mathrm{C}$ for $90 \mathrm{sec}$ followed by a final $10 \mathrm{~min}$ at $72^{\circ} \mathrm{C}$. PCR products were hybridized to digoxigenin (DIG)-labeled detection probes and immobilized via the biotin label to a streptavidin-coated microtiter plate. Detection was performed with a horseradish peroxidase conjugated antibody directed against DIG (AntiDIG-HRP) in a standard ELISA assay. A control template with a known amount of telomerase activity (provided with the kit) was used for determining the relative telomerase activity (RTA).

\section{Senescence assay}

MCF-7 cells were washed twice with PBS and fixed with $2 \%$ formaldehyde, $0.2 \%$ glutaraldehyde for $5 \mathrm{~min}$. The cells were then washed again with PBS and stained with a solution of $1 \mathrm{mg} / \mathrm{ml} 5$-bromo-4-chloro-3-inolyl- $\beta$ galactosidase in dimethylformamide (20 mg/ml stock), $5 \mathrm{mM}$ potassium ferrocyanide, $150 \mathrm{mM} \mathrm{NaCl}, 40 \mathrm{mM}$ citric acid/sodium phosphate, $\mathrm{pH} 6.0$, and $2 \mathrm{mM} \mathrm{MgCl} 2$. Following overnight incubation at $37^{\circ} \mathrm{C}$, the cells were washed twice with PBS and then photographed.

\section{ATP assay}

The level of ATP in MCF-7 cell lines was determined using the ATP Bioluminescence Assay Kit (Beyotime, China). Briefly, harvested cultured cells were lysed with a lysis buffer, followed by centrifugation at $10,000 \times \mathrm{g}$ for $2 \mathrm{~min}$ at $4^{\circ} \mathrm{C}$. The level of ATP was determined by mixing $50 \mu \mathrm{l}$ of the supernatant with $50 \mu \mathrm{l}$ of luciferase reagent, which catalyzed the light production from ATP and luciferin. The emitted light was linearly related to the ATP concentration and measured using a microplate luminometer.

\section{ROS assay}

The fluorescent probe, 2,7-dichlorofluorescin (DCFHDA, Sigma) was employed to quantify the level of ROS as described (Wan et al., 2005). Cells were prepared as described in Section 2.1. Confluent cultures were washed with D-Hank's buffer solution complemented with $1 \%$ FBS at $37^{\circ} \mathrm{C}$ for $2 \mathrm{~min}$, stained with $2 \mathrm{M}$ DCFH-DA for $40 \mathrm{~min}$ in incubator and then washed with cold D-Hank's twice. The fluorescence was measured in FDBS with fluorescence micro-plate reader $(485 / 520 \mathrm{~nm})$ and the values of relative fluorescence intensity were normalized to the controls.

\section{Cell cycle distribution analysis}

Cells were collected at different time points after treatments, washed twice with a cold phosphate buffered solution (PBS), fixed with $70 \%$ ethanol in PBS for 30 min, and then stained with a solution containing $5 \mu \mathrm{g} /$ $\mathrm{ml}$ propidium iodide (PI), $10 \mathrm{kU} / \mathrm{ml}$ RNase (Sigma), and $0.005 \%$ Triton $\mathrm{x}-100$ in the dark for $30 \mathrm{~min}$ at room temperature before the flow cytometric analysis. The samples were detected with FACS Calibur (Becton, Dickinson and Company, USA). A minimum of 10000 cells analyzed in each sample served to determine the percentages of cells in each phase of the cell cycle using Flowjo 7.2.1 software. Three independent experiments were performed.

\section{Semi quantitative and quantitative RT PCR}

The mRNA expression was determined by semi quantitative RT-PCR assays. Total RNA was extracted using FastPure RNA Kit (TaKaRa). First strand cDNA synthesis from $2 \mu \mathrm{g}$ of total RNA was performed using First-strand cDNA Synthesis System for Quantitative RT-PCR (Marligen Biosciences, Inc.). hTERT, hTR, and hTP mDNA were amplified by using specific primers. The sequence information of the primers was listed in Table 
1. PGC-1 $\alpha$ mRNA used specific primers as previously described (Bhalla et al., 2011) . $\beta$-actin mRNA used as an external standard was amplified from the same cDNA reaction mixture; The PCR program used consists of 10 $\min$ at $60^{\circ} \mathrm{C}$ for reverse transcription, $30 \mathrm{sec}$ at $95^{\circ} \mathrm{C}$ followed by 40 cycles of denaturation $\left(95^{\circ} \mathrm{C}\right)$, annealing $\left(10 \mathrm{sec}\right.$ at $\left.60^{\circ} \mathrm{C}\right)$ and enzymatic chain extension (10 sec at $72^{\circ} \mathrm{C}$ ). Amplified products were electrophoresed on a $2 \%$ agarose gel and stained with ethidium bromide. The quantitative RT PCR was carried out in FTC-3000 qPCR system (FUNGLYN, Canada) with the same primers and conditions described above. Triplicate reactions were performed for each marker in $50 \mu$ l tubes using a three-step amplification program of 40 cycles of $95^{\circ} \mathrm{C}$ for $5 \mathrm{~s}, 60^{\circ} \mathrm{C}$ for $10 \mathrm{~s}$ and $72^{\circ} \mathrm{C}$ for $10 \mathrm{~s}$. Relative mRNA expression was calculated as the ratio of the amplification amount obtained with each marker versus $\beta$-actin for each sample and plotted normalized to the control group.

\section{DNA isolation and purification}

Total DNA was purified using DNA Blood and Tissue Kit (Tiangen) from cells with/without ionizing radiation, respectively, and DNA quantity and purity was determined by spectrometric analysis. The isolated DNA showed a high purity $(\mathrm{A} 260 / \mathrm{A} 280>1.8)$ and was stored at $4^{\circ} \mathrm{C}$ according to standard procedures.

\section{MtDNA copy number quantification}

Total genomic DNA of $50 \mathrm{ng}$ were used for mtDNA and nuclear DNA markers, respectively, in a $20 \mu \mathrm{l}$ reaction containing $1 \mathrm{X}$ SYBR Premix Ex Taq II (TaKaRa, Japan) and $200 \mathrm{nmol} / \mathrm{l}$ each primer. The sequence information regarding the primers was as follow: ND1: CACCCAAGAACAGGGTTTGT and TGGCCATGGGTATGT T G T TA A ; $\beta$-actin: CAAAACCTAACTTGCGCAGA and TTTTAGGATGGCAAGGGACT. Triplicate reactions were performed for each marker in $50 \mu$ l tubes using a three-step amplification program of 40 cycles of $95^{\circ} \mathrm{C}$ for $5 \mathrm{~s}, 55^{\circ} \mathrm{C}$ for $10 \mathrm{~s}$ and $72{ }^{\circ} \mathrm{C}$ for $10 \mathrm{~s}$. Standard curves were generated in each reaction using serial dilutions of untreated genomic DNA. Relative mtDNA copy number was calculated as the ratio of the amplification amount obtained with mtDNA markers versus $\beta$-actin for each sample and plotted normalized to the control group.

\section{Long-run quantitative PCR for mtDNA damage assay}

Long-run quantitative PCR was carried out as our previous study (Zhou etal.,2012). The $8.9 \mathrm{~kb}$ amplicons from Santos and colleagues (Santos et al., 2006a) was used for quantification of mtDNA damage. The primer information was listed in supplement as follow: Long fragment 599914841: TCTAAGCCTCCTTATTCGAGCCGA and TTTCATCATGCGGAGATGTTGGATGG; short fragment 14620-14841: CCCCACAAACCCCATTACTAAACCCA and TTTCATCATGCGGAGATGTTGGATGG. The experiment procedures were carried out according to the protocol of Santos et.al (Santos et al., 2006a).

Mitochondrial mass and mitochondrial membrane potential measurements
The fluorescent probe, MitoTracker Green FM (Invitrogen, USA) was employed to measure the relative amount of mitochondrial mass and JC-1 (Sigma, USA) staining was used to indicate the mitochondrial membrane potential changes. Mitochondrial mass were measured by staining with Mito Tracker Green FM $(60 \mathrm{nM})$ for $25 \mathrm{~min}$. Unstained cells were analyzed as controls and used to gate on live cells for final analysis. The samples were detected with FACS Calibur (Becton, Dickinson and Company, USA). A minimum of 10,000 cells analyzed in each sample served to determine the amount of mitochondrial mass using Flowjo 7.2.1 software. Three independent experiments were performed. For mitochondrial membrane potential measurement, MCF-7 cells were resuspended after trypsinization in $1 \mathrm{ml}$ of medium and incubated with $10 \mu \mathrm{g} / \mathrm{ml}$ of $\mathrm{JC}-1$ at $37^{\circ} \mathrm{C}, 5 \% \mathrm{CO}_{2}$, for $30 \mathrm{~min}$. Both red and green fluorescence emissions were analyzed by flow cytometry after JC-1 staining. The average ratio of $525 \mathrm{~nm} / 570 \mathrm{~nm}$ in at least 10,000 cells was quantified by Flowjo 7.2.1 software.

\section{Immunoblotting}

Western blotting protocol followed standard protocol. Briefly, MCF-7 cells were lysed in buffer containing 50 $\mathrm{mmol} / \mathrm{l}$ Tris at $\mathrm{pH} 7.4,50 \mathrm{mmol} / \mathrm{l} \mathrm{NaCl}, 0.1 \%$ Triton $\mathrm{X}-100,0.1 \% \mathrm{SDS}, 0.3 \mathrm{mmol} / \mathrm{l}$ sodium orthovanadate, $1 \mathrm{mmol} / \mathrm{l}$ dithiotheritol, $10 \mathrm{mg} / \mathrm{l}$ leupeptin, and $5 \mathrm{mg} / \mathrm{l}$ aprotinin. Protein concentrations of cell lysates were determined using a BCA protein assay kit (Pierce, Rockford, IL, USA). An aliquot of each extract (40 $\mu \mathrm{g}$ protein) was fractionated by electrophoresis in an SDSpolyacrylamide gel and transferred to a PVDF membrane. Membranes were blocked with $10 \mathrm{ml}$ TBST containing 0.5 $\mathrm{g}$ FBS at room temperature for $2 \mathrm{~h}$, followed by incubation with antibodies against hTERT, PGC- $1 \alpha$ and $\beta$-actin (Abcam, USA) at $4{ }^{\circ} \mathrm{C}$ overnight. After washing with TBST for $30 \mathrm{~min}$, appropriate HRP-conjugated secondary antibody was added to the membranes, which were incubated at room temperature for $1 \mathrm{~h}$. Membranes were washed three times for 15 min each with TBST. Reactive proteins were visualized using a chemiluminescence kit (Santa Cruz Biotechnology, Santa Cruz, CA) according to the manufacturer's instructions.

\section{Statistical analysis}

Statistical analysis was performed on the means of the data obtained from at least three independent experiments. Data are presented as means \pm SD. Student's t-test program in Microsoft Excel was used to detect statistical significance. $p<0.05$ was considered to be statistically significant.

\section{Results}

Telomerase activity was suppressed by carbon-ion irradiation via hTERT down-regulation

To find out whether telomere function was related to radiosensitivity in response to carbon-ion irradiation, telomerase activity was measured after irradiation. As shown in Figure 1A, telomerase activity was suppressed after $4 \mathrm{~Gy}$ and $10 \mathrm{~Gy}$ carbon-ion irradiation. Three of 


\section{Guo-Ying Miao et al}

the telomerase subunits, which are hTERT, hTR and hTP were screened for their mRNA expression before and after carbon-ion irradiation. No significant changes in hTP and hTR were detected, while hTERT mRNA expression was markedly suppressed within 48 hours after 4 Gy and 10 Gy carbon-ion irradiation (Figure 1B). The down-regulation of hTERT expression was further confirmed by real-time RT PCR in mRNA level (Figure 1C) and western blot in protein expression level (Figure 1D). Since hTERT is the catalytic subunit of telomerase, it's likely that carbon-ion radiation could inhibit telomerase activity via hTERT suppression.

Telomerase inhibition led to increased radiosensitivity and cell cycle redistribution

To confirm that hTERT activity contributes to the carbon-ion radiation induced cellular inactivation, we further testified whether hTERT inhibition could lead to enhanced radiosensitivity. MCF-7 incubated with $2 \mu \mathrm{M}$ hTERT inhibitor MST-312 exhibited significantly reduced clonal survival after 2-4 Gy carbon-ion irradiation as compared to irradiation alone (Figure 2). 2 Gy carbon-

\section{Table 1. DNA Primers Used for PCR}

\begin{tabular}{|c|c|}
\hline \multirow{2}{*}{ hTERT } & 5' - CGGAAGAGTGTCTGGAGCAA-3' \\
\hline & 5'- GGATGAAGCGGAGTCTGGA-3' \\
\hline \multirow{2}{*}{ hTR } & 5'-TCAGACAGTCCGGGACATCAT-3' \\
\hline & 5'- CCTTCCTTCTATTTGTCGGTCG-3' \\
\hline \multirow{2}{*}{ hTP } & 5'-GAAGGGCGTAGGCGCCGTGCTTTTGC-3' \\
\hline & 5'-GTTTGCTCTAGAATGAACGGTGGAAGG-3' \\
\hline \multirow{2}{*}{ PGC- $1 \alpha$} & 5' - AACAGCAGCAGAGACAAATGCACC-3' \\
\hline & 5'-TGCAGTTCCAGAGAGTTCCACACT-3' \\
\hline \multirow{2}{*}{$\beta$-actin } & 5'-TTGTTACCAACTGGGGACGATA-3 ’ \\
\hline & 5'-TCGTCCTTCTAGTTCTAG-3' \\
\hline
\end{tabular}

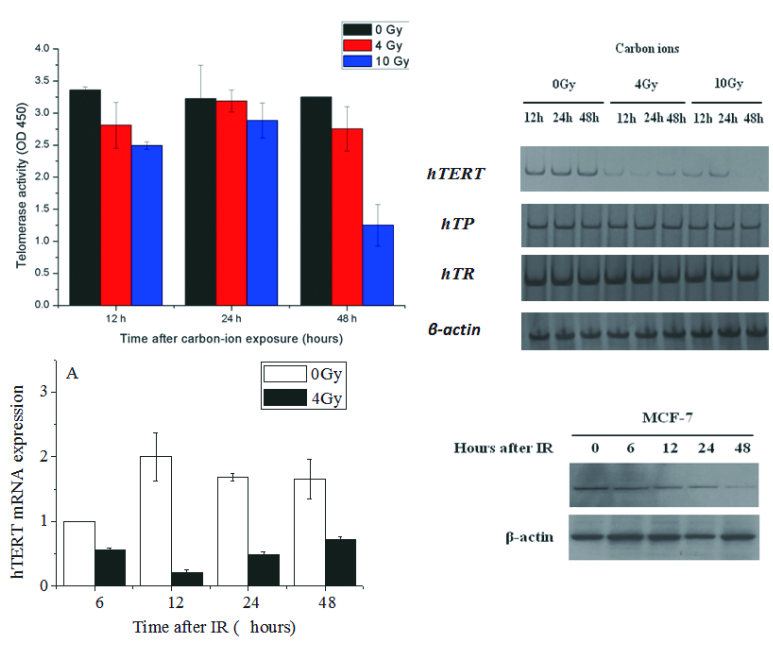

Figure 1. Carbon-Ion Irradiation Depressed Telomerase Activity via hTERT Downregulation. A. Telomerase activity in treated MCF-7 cells. B. RT-PCR analysis of expression of hTERT, TP-1, and hTR mRNA in MCF-7 cell line. Actin expression was used as controls for RNA loading and RT efficiency. C. Real-time RT-PCR confirmation of hTERT expression in MCF-7 cells after carbon-ion irradiation. D. Western blot for hTERT protein expression. *: $\mathrm{p}<0.05$ versus control ion irradiation caused $46 \%$ clonal survival versus control. Clonal survival further reduced to $17 \%$ with the addition of MST-312 (Figure 2A). Carbon-ion irradiation alone induced mostly G2-M cell cycle arrest (Figure 2B). G2-M phase arrest was pronounced 24 hours after carbon-ion irradiation. The G2-M arrest was alleviated in the subsequent 48 hours but was still elevated versus control. G2-M arrest shifted to G1-S phase arrest with the MST-312 incubation (Figure 2B, bottom panel). With the addition of MST-312, irradiated cells exhibited $\mathrm{S}$ phrase arrest instead of G2-M arrest. Cell population in G1-S phase was about two fold compare to irradiation alone $(27.35 \%$ vs $14.69 \%)$, the increase proportion of G1-S phase cells continued in the later 48 hours. This was expectable because telomerase synthesized telomere during S phase. With telomerase inhibition by MST312, cells may not pass cell cycle check point in $\mathrm{S}$ phase.

\section{hTERT suppression impaired mitochondrial function}

The above results indicated that telomerase was closely related to radiosensitivity of MCF-7 to carbonion irradiation. The functional subunit hTERT could be suppressed by either carbon-ion or MST-312. For further understanding the signaling pathways regulating hTERT mediated cellular inactivation, its down-stream effectors should be determined. Mitochondria have been reported to be a potential down-stream effecter of HTERT via p53-PGC-1 $\alpha$ pathway (Sahin et al., 2011). Since MCF7 has wild-type p53, the proposed telomere-p53-PGC axis may also apply to our current system. Indeed we found that either MST-312 or carbon-ion radiation could
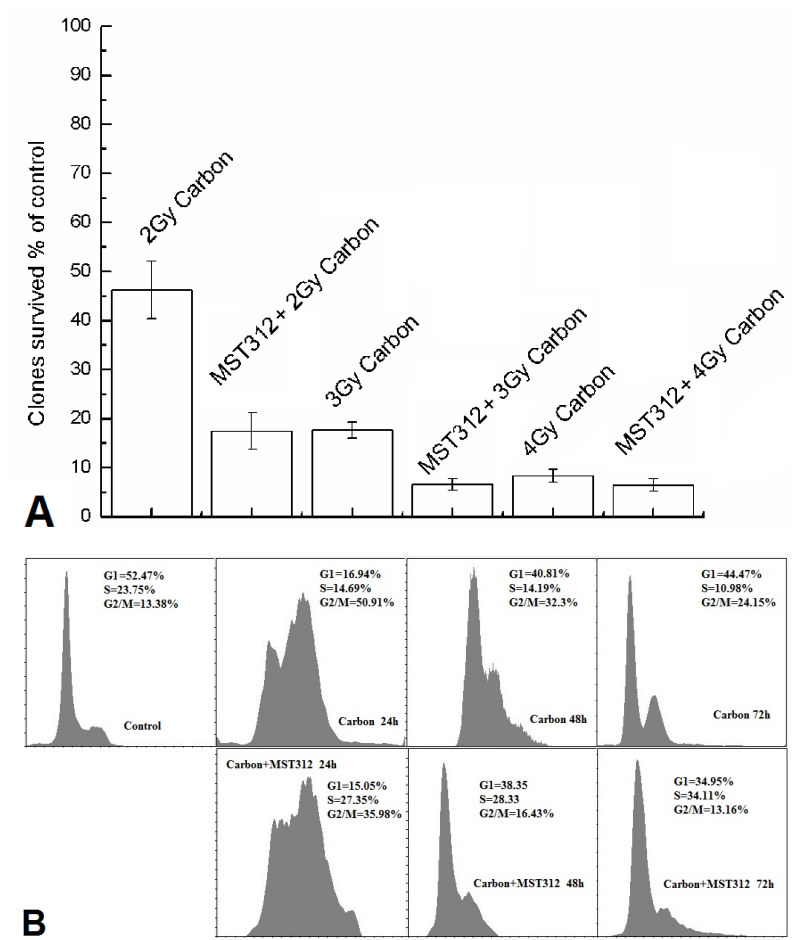

Figure 2. Inhibition of hTERT Sensitize MCF-7 Cells to Carbon-Ion Irradiation. A. Radiosensitivity of MCF-7 cells as measured by clone survival after carbon-ion irradiation. B. Cell cycle redistribution after carbon-ion irradiation in MCF-7 cells with/without MST-312 treatment 
induce mitochondria dysfunction. Carbon-ion irradiation caused reduced ATP generation, elevated ROS generation, decreased mitochondrial membrane potential and mtDNA copy number (Figure 3A). For further confirmation, MCF-7 cells incubated with MST-312 were used for these assays. Again we found that all the parameters followed the same pattern (Figure 3B). These data suggest that telomere dysfunction, either caused by carbon-ion irradiation or direct MST-312 inhibition, could lead to mitochondrial dysfunction, which is consistent with other reports (Sahin et al., 2011; David, 2011; Wenz, 2013).

Mitochondrial ROS contributed to MCF-7 cells senescence after carbon-ion irradiation

Our previous study showed that MCF-7 cells

A

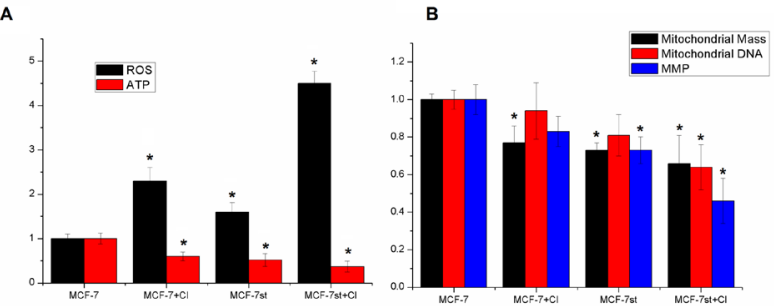

Figure 3. Telomere Dysfunction Impaired Mitochondrial Biogenesis and Redox Status in MCF-7 Cells Or Cells that Incubated Continuously with MST with MST-312 (MCF-7st) Before/After Carbon-Ion Irradiation (CI). A. Cellular energy and redox state in MCF-7 and MCF-7st cells after carbon-ion irradiation. ATP production was measured using commercial available kit; ROS level was detected using DCFHDA staining. B. Effects of telomere dysfunction and carbon-ion irradiation on mitochondrial biogenesis. MtDNA copy number was measured by real-time PCR; Mitochondrial mass and mitochondrial membrane potential (MMP) were quantified by FACS using MitoTracker green and JC-1 staining, respectively, $*$ : $\mathrm{p}<0.05$ versus control

A

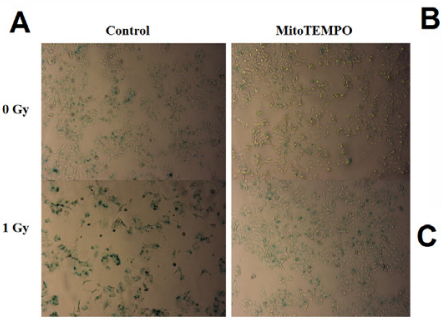

B
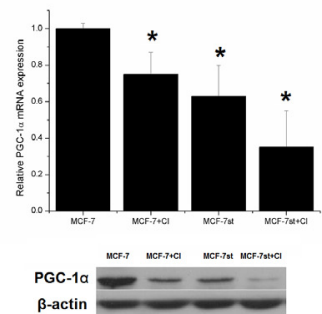

Figure 4. Mitochondrial ROS Contributed to MCF7 Cells Senescence After Carbon-Ion Irradiation. A. Representative image of cellular senescence after $1 \mathrm{~Gy}$ carbon-ion irradiation. Senescent cells were evident in MCF7 cells incubated continuously with MST-312 (MCF-7st). Cellular senescence was further aggravated by 1 Gy carbonion radiation. MCF-7st cells were continuously cultured with the presence of $5 \mu \mathrm{M}$ mitochondrial antioxidant MitoTEMPO. MitoTEMPO not only partially prevented irradiated MCF-7st cells from entering senescence, but also reduced the basal level of cellular senescence. B. Regulation of PGC- $1 \alpha$ in MCF-7 cells in transcription levels before/after carbon-ion irradiation. Transcription of PGC-1 $\alpha$ mRNA was quantified by real-time RT-PCR; C. Representative image of western blotting for PGC$1 \alpha$ expression in MCF-7 cells after carbon-ion irradiation. *: $\mathrm{p}<0.05$ versus control inactivation induced by carbon-ion irradiation was mainly caused by senescence. To verify whether mitochondrial dysfunction contributes to the senescence progress in MCF-7 cells after carbon-ion radiation, MitoTEMPO was used to relieve the effect of mitochondrial dysfunction. As illustrated in Figure 4A, MitoTEMPO not only partially prevented irradiated MCF-7 cells from entering senescence, but also reduced the basal level of cellular senescence at the presence of MST-312. PGC- $1 \alpha$ is the master switch of mitochondrial biogenesis. It's therefore logical to determine whether mitochondrial dysfunction was regulated by PGC- $1 \alpha$ Expression. PGC- $1 \alpha$ was repressed at both transcription and translation level in MCF-7 cells incubated with MST-312. Carbon-ion radiation could lead to further PGC- $1 \alpha$ repression (Figure 4B and C).

\section{Discussion}

TERT is the catalytic subunit of telomerase. Besides its core function in telomere synthesis, it is also localized to mitochondria. Sharma et al demonstrate that mitochondrial hTERT works as a hTR-independent reverse transcriptase, which has different cellular functions in nucleus and mitochondria (Sharma et al., 2012). Our previous finding showed that the mean telomere length was not statistically changed in human breast cancer cells MCF-7 within 10 days post carbon-ion irradiation (Zhou et al., 2013), which is consist with the observation in human gliomaderived cell line NP-2 10 days after carbon-ion irradiation (Jinno-Oue et al., 2010). Here we find that carbon-ion irradiation suppressed telomerase activity, and hTERT was down-regulation but not hTR nor TP-1, while telomere length was not decreased (data not shown). These results indicate that the mitochondria-related function of hTERT rather than that in nucleus could play an important role in carbon-ion-induced senescence.

TERT has been found to be excluded from nucleus upon oxidative stress and localize in the mitochondria (Santos et al., 2004). Shaheda et al. found that the mitochondrial localization of hTERT improve mitochondrial function, including reduced mtDNA damage, lower mitochondrial ROS production and enhanced MMP (Ahmed et al., 2008). Recent study showed that upregulation of mitochondrial hTERT counteracts the chemotherapeutic effects of cisplatin and 5-fluorouracil, as both mitochondrial ROS and mtDNA damage were decreased (Yan et al., 2015). Conversely, the decreased overall hTERT expression, either caused by carbon-ion irradiation or MST-312 incubation, could be one of the causes of mitochondrial dysfunction, including decreased mtDNA copy number, increased mtDNA damage and impaired mitochondrial function in our study.

Mitochondrial biogenesis in breast cancer cells, such as MCF-7, has been proved to play a crucial role in response to cellular stress. Promoting mitochondrial function can confer tamoxifen-resistance in MCF-7 cells (Martinez-Outschoorn et al., 2011a; Martinez-Outschoorn et al., 2011b). Conversely, mitochondrial dysfunction induced by tamoxifen plus metformin could reverse this drug-resistance (Martinez-Outschoorn et al., 2011a). This 
is true since addition of a mitochondrial ROS scavenger MitoTEMPO could alleviate the extent of cellular senescence induced by hTERT inhibition and carbon-ion irradiation in our study.

In summary, our current findings showed that telomere-mitochondria axis regulated the cellular inactivation process in MCF-7 cells. Targeting telomere and its downstream effecter mitochondria could be a potential way for further effectiveness improvement of carbon-ion radiotherapy.

\section{Acknowledgements}

The authors would like to thank External Cooperation Program of Chinese Academy of Sciences, Japan Society for the Promotion of Science and National for Cooperative Research Program, the Key program of National Natural Science Foundation of China (U1432248) and the National Natural Science Foundation of China (11405230 and 11505245). The authors also thank Dr. K.Tanaka, Dr. T. Katsube, Dr. Bing Wang, Ms. H. Arai and Ms. M. Nakajima for their technical assistance throughout the study.

\section{References}

Ahmed S, Passos J F, Birket M J, et al (2008). Telomerase does not counteract telomere shortening but protects mitochondrial function under oxidative stress, J Cell Sci, 121, 1046-53.

Bhalla K, Hwang BJ, Dewi RE, et al (2011). PGC1alpha promotes tumor growth by inducing gene expression programs supporting lipogenesis. Cancer Res, 71, 6888-98

Buchner N, Zschauer TC, Lukosz M, Altschmied J, Haendeler J (2010). Downregulation of mitochondrial telomerase reverse transcriptase induced by $\mathrm{H}_{2} \mathrm{O}_{2}$ is $\mathrm{Src}$ kinase dependent. Experimental Gerontol, 45, 558-62.

David R (2011). Ageing: Mitochondria and telomeres come together Nature reviews. Molecular Cell Biol, 12, 204.

Durante M, George K and Cucinotta EA (2006). Chromosomes lacking telomeres are present in the progeny of human lymphocytes exposed to heavy ions. Radiat Res, 165, 51-8.

Gordon DM, Santos JH (2010). The emerging role of telomerase reverse transcriptase in mitochondrial DNA metabolism. $J$ Nucleic Acids, 2010.

Haendeler J, Drose S, Buchner N, et al (2009). Mitochondrial telomerase reverse transcriptase binds to and protects mitochondrial DNA and function from damage. Arterioscler Thromb Vasc Biol, 29, 929-35.

Indran IR, Hande MP, Pervaiz S (2011). hTERT overexpression alleviates intracellular ROS production, improves mitochondrial function, and inhibits ROS-mediated apoptosis in cancer cells. Cancer Res, 71, 266-76.

Jakel O, Karger CP, Debus J (2008). The future of heavy ion radiotherapy. Medical Physics, 35, 5653-63.

Jinno-Oue A, Shimizu N, Hamada N, et al (2010). Irradiation with carbon ion beams induces apoptosis, autophagy, and cellular senescence in a human glioma-derived cell line Int J Radiat Oncol Biol Phys, 76, 229-41.

Lee YH, Kang BS, Bae YS (2014). Premature senescence in human breast cancer and colon cancer cells by tamoxifenmediated reactive oxygen species generation. Life Sci, $\mathbf{9 7}$, 116-22.

Li YB, Gao JL, Zhong ZF, et al (2013). Bisdemethoxycurcumin suppresses MCF-7 cells proliferation by inducing ROS accumulation and modulating senescence-related pathways Pharmacological Reports, 65, 700-9.

Liu L, Trimarchi JR, Smith PJ, Keefe DL (2002). Mitochondrial dysfunction leads to telomere attrition and genomic instability. Aging Cell, 1, 40-6.

Martinez-Outschoorn UE, Goldberg A, Lin Z, et al (2011a). Anti-estrogen resistance in breast cancer is induced by the tumor microenvironment and can be overcome by inhibiting mitochondrial function in epithelial cancer cells. Cancer Biol Ther, 12, 924-38.

Martinez-Outschoorn UE, Lin Z, Ko YH, et al (2011b). Understanding the metabolic basis of drug resistance: therapeutic induction of the Warburg effect kills cancer cells Cell Cycle, 10, 2521-8.

Sahin E, Colla S, Liesa M, et al (2011). Telomere dysfunction induces metabolic and mitochondrial compromise. Nature, 470, 359-65.

Santos JH, Meyer JN, Mandavilli BS, Van Houten B (2006a). Quantitative PCR-based measurement of nuclear and mitochondrial DNA damage and repair in mammalian cells. Methods Mol Biol, 314, 183-99.

Santos JH, Meyer JN, Skorvaga M, Annab LA, Van Houten B (2004). Mitochondrial hTERT exacerbates free-radicalmediated mtDNA damage. Aging Cell, 3, 399-411.

Santos JH, Meyer JN, Van Houten B (2006b). Mitochondrial localization of telomerase as a determinant for hydrogen peroxide-induced mitochondrial DNA damage and apoptosis. Hum Mol Genet, 15, 1757-68.

Saretzki G (2009). Telomerase, mitochondria and oxidative stress Experimental Gerontol, 44, 485-92.

Sharma NK, Reyes A, Green $P$, et al (2012). Human telomerase acts as a hTR-independent reverse transcriptase in mitochondria. Nucleic Acids Res, 40, 712-25.

Wan XS, Zhou Z, Ware JH, Kennedy AR (2005). Standardization of a fluorometric assay for measuring oxidative stress in irradiated cells. Radiation Res, 163, 232-40.

Wenz T (2013) Regulation of mitochondrial biogenesis and PGC1 alpha under cellular stress. Mitochondrion, 13, 134-42.

Yan J, Zhou Y, Chen D, et al (2015). Effects of mitochondrial translocation of telomerase on drug resistance in hepatocellular carcinoma cells. J Cancer, 6, 151-9.

Zhou X, Liu X, Zhang X, et al (2012). Non-randomized mtDNA damage after ionizing radiation via charge transport. Sci Rep, $2,780$.

Zhou X, Zhang X, Xie Y, et al (2013). DNA-PKcs inhibition sensitizes cancer cells to carbon-ion irradiation via telomere capping disruption. PLoS One, 8, 72641. 